\title{
Asymptomatic Gangrenous Cholecystitis Discovered During Elective Laparoscopic Cholecystectomy
}

\author{
Darren Porter ${ }^{\mathrm{a}, \mathrm{b}}$, Conor Warren ${ }^{\mathrm{a}}$, Hisham Hurreiz ${ }^{\mathrm{a}}$
}

\begin{abstract}
Gangrenous cholecystitis (GC) is a serious complication of acute cholecystitis. It is associated with significant morbidity and mortality. A male with type 2 diabetes mellitus had been treated for acute cholecystitis and discharged home. The patient was admitted electively for a laparoscopic cholecystectomy 6 weeks post discharge at which time he was entirely asymptomatic. Intra-operatively the gallbladder was found to be necrotic but it was removed laparoscopically and without complication. Acute cholecystitis is the most common complication of cholelithiasis occurring in approximately $1-2 \%$ of asymptomatic patients annually; however, in approximately $2-29.6 \%$ of patients, the disease processes to GC. GC is associated with a significant morbidity and mortality; however, in spite of its grave prognosis, its diagnosis can be elusive both clinically and biochemically. Many factors have been implicated in the formation of GC, including diabetes mellitus, age $\geq 51$ years, male sex, leucocytosis $\left(\mathrm{WCC}>15 \times 10^{9}\right)$ and a high CRP. Complications associated with GC include perforation, which has been reported to occur in up to $10 \%$ of cases of acute cholecystitis. When managing a patient with acute cholecystitis, a high index of suspicion is essential for the possibility of GC, especially in a diabetic and elderly patient even if the patient appears to be improving clinically and in the presence of normalizing laboratory investigations. There is a need to consider early or emergency cholecystectomy in these patients due to the high risk of gangrenous transformation with possibility of gallbladder perforation and a resultant increased morbidity and mortality.
\end{abstract}

Keywords: Gangrenous cholecystitis; Asymptomatic; Increased morbidity and mortality; Early cholecystectomy

Manuscript accepted for publication October 18, 2013

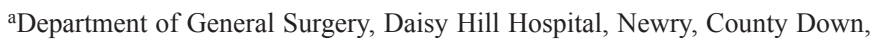
BT35 8DR, UK

${ }^{\mathrm{b} C}$ Corresponding Author: Darren Porter, 49 Diamond Gardens, Finaghy, Belfast, BT10 0HE, County Antrim, Northern Ireland, UK. Email: dporter@tcd.ie

doi: http://dx.doi.org/10.14740/jmc1530w

\section{Introduction}

Gangrenous cholecystitis (GC) is a serious complication of acute cholecystitis [1]. The incidence of GC varies from $2 \%$ to $29.6 \%$ of all cases of acute cholecystitis and is associated with significantly greater morbidity and mortality [2].

Risk factors for GC include diabetes mellitus, advanced age, male sex, leucocytosis, history of coronary artery disease, critical illness and a high C-reactive protein (CRP) [3].

Forty-eight percent of patients with histologically proven GC have little or no evidence to suggest this disease process pre-operatively. Once suspected, patients with GC should undergo urgent or emergency cholecystectomy in order to avoid potentially life-threatening complications [4].

\section{Case Report}

A 50-year-old Caucasian male with a known history of type 2 diabetes mellitus was admitted with severe right upper quadrant pain of 3 days duration, which worsened on the day of admission and was associated with nausea, anorexia and fever.

On initial examination, the patient had a sinus tachycardia of $113 \mathrm{bpm}$, observations were otherwise normal and he was apyrexic. The patient was markedly tender over the right upper quadrant and Murphy's sign was positive. Laboratory investigations revealed a leucocytosis of $17.8 \times 10^{9} / \mathrm{L}$, a CRP of $80 \mathrm{mg} / \mathrm{L}$, a bilirubin of 12.4, a Gamma-glutamyltransferase of 12 , an alkaline phosphatase of $69 \mathrm{IU} / \mathrm{L}$, and an amylase of 35. The patient was diagnosed with acute cholecystitis and commenced on IV co-amoxiclav $1.2 \mathrm{~g}$ TID.

An ultrasound abdomen performed on the morning after admission demonstrated calculi within the gallbladder, a thickened gallbladder wall with pericholecystic fluid but no common bile duct dilatation.

The patient completed a 5-day course of IV co-amoxiclav with complete resolution of his symptoms and he was discharged home with normal inflammatory markers to have a laparoscopic cholecystectomy at a later date.

An elective laparoscopic cholecystectomy was performed 6 weeks after discharge, by which time the patient was asymptomatic and was systemically well. Admission observations were normal as were pre-operative bloods. 


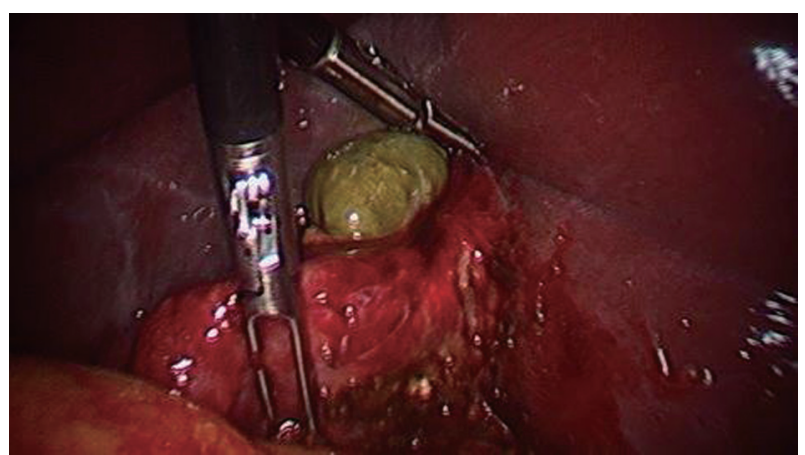

Figure 1. Necrotic gallbladder fundus demonstrated intra-operatively.

During the laparoscopy, the fundus of the gallbladder was found to be gangrenous and covered by adherent omentum. The omenal adhesions were gently freed revealing an entirely necrotic gallbladder fundus (Fig. 1). The cystic duct and artery were identified and clipped and the gallbladder dissected off the liver bed. The procedure was completed laparoscopically without complication. The patient was discharged home on day 1 post-operatively on a 5-day course of oral co-amoxiclav following advice from the microbiology department.

\section{Discussion}

Acute cholecystitis is the most common complication of cholelithiasis occurring in approximately 1-2\% of asymptomatic patients annually [5].

The primary etiology of acute cholecystitis is obstruction of the cystic duct secondary to an impacted stone. In approximately $80 \%$ of patients, the impacted stone dislodges allowing for initial conservative non-operative management. However, in approximately $2-29.6 \%$ of patients, the disease processes to GC.

The etiology of GC is primarily vascular compromise secondary to sustained obstruction of the cystic duct. The persistent obstruction of the cystic duct results in marked distension of the gallbladder with increased tension in the gallbladder wall and resultant epithelial injury. As a result of the epithelial injury, phospholipases are released that degrade adjacent cell membranes leading to an intense inflammatory reaction. It is the combination of increased gallbladder wall tension and the intense inflammatory reaction that results in either local or global gallbladder wall ischemia and subsequently to ischemic necrosis of the gallbladder wall [6].

$\mathrm{GC}$ is associated with a significantly greater morbidity and mortality when compared to uncomplicated acute cholecystitis [7]. In spite of its grave prognosis, the diagnosis of GC can be elusive, both clinically and biochemically, with $48 \%$ of patients demonstrating histologically proven GC displaying no evidence to suggest this disease process pre-operatively. It is often only considered after deterioration of a patient suspected of having uncomplicated cholecystitis.

Many factors have been implicated in the formation of $\mathrm{GC}$, including diabetes mellitus, age $\geq 51$ years, male sex, leu- cocytosis (WCC $>15 \times 10^{9}$ ), history of coronary artery disease; other associated risk factors include critical illness and a high CRP.

Due to the associated increased morbidity and mortality, several authors have advocated emergency cholecystectomy for GC. There is controversy regarding the best surgical approach for GC. Some surgeons would opt for open cholecystectomy for most men over the age of 60 years who have significant comorbidity, large gallstones and a raised bilirubin level; however, in the hands of an experienced laparoscopic surgeon, laparoscopic cholecystectomy can be used safely and successfully in patients with GC [7].

Complications associated with GC include perforation, which has been reported to occur in up to $10 \%$ of cases of acute cholecystitis. It is therefore vital to diagnose and surgically treat GC prior to perforation to avoid the associated morbidity and mortality.

\section{Conclusion}

When managing a patient with acute cholecystitis, a high index of suspicion is essential for the possibility of GC, especially in a diabetic and elderly patient even if the patient appears to be improving clinically and in the presence of normalising laboratory investigations. Radiological investigations may not conclusively demonstrate GC.

There is a need to consider early or emergency cholecystectomy (open or laparoscopic) in these patients due to the high risk of gangrenous transformation with possibility of gallbladder perforation and a resultant increased morbidity and mortality.

\section{Grant}

Not applicable.

\section{Conflicts of Interest}

We have no conflicts of interest to declare.

\section{References}

1. Bingener J, Stefanidis D, Richards ML, Schwesinger WH, Sirinek KR. Early conversion for gangrenous cholecystitis: impact on outcome. Surg Endosc. 2005;19(8):11391141.

2. Hunt DR, Chu FC. Gangrenous cholecystitis in the laparoscopic era. Aust N Z J Surg. 2000;70(6):428-430.

3. Merriam LT, Kanaan SA, Dawes LG, Angelos P, Prystowsky JB, Rege RV, Joehl RJ. Gangrenous cholecystitis: analysis of risk factors and experience with laparoscopic cholecystectomy. Surgery. 1999;126(4):680-685; discussion 685-686.

4. Teefey SA, Baron RL, Radke HM, Bigler SA. Gangre- 
nous cholecystitis: new observations on sonography. J Ultrasound Med. 1991;10(11):603-606.

5. Friedman GD. Natural history of asymptomatic and symptomatic gallstones. Am J Surg. 1993;165(4):399404.

6. Croley GG, 2nd. Gangrenous cholecystitis: five patients with intestinal obstruction. Am Surg. 1992;58(5):284292.

7. Habib FA, Kolachalam RB, Khilnani R, Preventza O, Mittal VK. Role of laparoscopic cholecystectomy in the management of gangrenous cholecystitis. Am J Surg. 2001;181(1):71-75. 